\title{
Invarianza factorial del Cognitive Emotional Regulation Questionnarie (CERQ) en universitarios limeños y cordobeses*
}

\author{
Factorial invariance of Cognitive Emotional Regulation Questionnaire \\ (CERQ) in college students from Lima and Cordoba
}

Recibido: 09 de abril de 2015 | Aceptado: 17 de septiembre de 2015

\author{
Sergio AleXis Dominguez LaRA** \\ Universidad de San Martín de Porres, Perú \\ LEONARDO ADRIÁN MEDRANO *** \\ Universidad Siglo 21, Argentina
}

doi: 10.11144/Javeriana.upsy15-1.ifce

Para citar este artículo: Dominguez-Lara, S., \& Medrano, L., (2016). Invarianza factorial del Cognitive Emotional Regulation Questionnarie (CERQ) en universitarios limeños y cordobeses. Universitas Psychologica, 15(1), 89-98. http://dx.doi.org/10.11144/ Javeriana.upsy15-1.ifce

* Artículo de investigación. La recopilación de información fue realizada durante la estancia del primer autor en la Universidad Inca Garcilaso de la Vega. Financiado por la Universidad Inca Garcilaso de la Vega (Lima, Perú)

** Correo electrónico: sdominguezl@usmp.pe; sdominguezmpcs@gmail.com

**** Correo electrónico: leomedpsico@gmail.com

\section{RESUMEN}

El objetivo de este trabajo fue el estudio de la invarianza factorial del Cognitive Emotional Regulation Questionnarie (CERQ), entre universitarios limeños (Perú) y cordobeses (Argentina), a fin de realizar una comparación intercultural de las estrategias cognitivas de regulación emocional. Se contó con la participación de 345 estudiantes universitarios limeños (hombres $=26.4 \%$; mujeres $=73.6 \%$ ), y 358 estudiantes universitarios de Córdoba (hombres $=49.7 \%$; mujeres $=49.7 \%$ ). Se realizó la evaluación progresiva de la invarianza factorial (configural, métrica, fuerte y estricta). Los resultados indican con relación a la invarianza configural que se verifica la estructura de nueve factores oblicuos, aunque un análisis pormenorizado y más restrictivo permite identificar un funcionamiento no-invariante en algunos reactivos e interceptos. Se concluye que las evidencias recabadas no son suficientes para elaborar un modelo explicativo de las diferencias observadas, ante lo cual podría indagarse en un futuro estudio si el uso de estas estrategias se relaciona con especificidades en las ideologías y valores de cada cultura. Palabras clave

regulación emocional; estudiantes universitarios; estudio intercultural; invarianza factorial

\section{A B S T R A C T}

The aim of this paper was to study the factorial invariance of Cognitive Emotional Regulation questionnarie (CERQ) among college students from Lima (Peru) and Cordoba (Argentina), to perform a cross-cultural comparison of cognitive emotion regulation strategies. 345 college students from Lima $(\mathrm{men}=26.4 \%$; women $=73.6 \%)$ and 358 college students from Córdoba $($ men $=49.7 \%$; women $=49.7 \%$ ) were examined. Progressive assessment of the factorial invariance (configural, metric, strong and strict) was performed. The results indicate regarding to configural invariance, the structure invariance of nine oblique factors was verified, and although a detailed analysis identifies a non-invariant functioning in some reactive and intercepts. We conclude that the collected evidence is not enough to develop a model to explain the observed differences, whereupon could ask about it in a future study whether the use of these strategies are relate to specific ideologies and values of each culture.

Keywords

emotional regulation; college students; intercultural study; factorial invariance 


\section{La regulación emocional y su medición}

En términos amplios la regulación emocional puede ser definida como "toda estrategia dirigida a mantener, aumentar o suprimir un estado afectivo en curso" (Thompson, 1994, p. 27). Es decir, que al experimentar un estado emocional (sobre todo displacentero) las personas despliegan una serie de conductas intencionadas y/o automáticas, que tienen como objetivo ejercer algún tipo de dominio sobre las emociones, ya sea modificando su intensidad o valencia (Koole, 2009).

La existencia de fallas en estos procesos de regulación emocional explicaría en parte la aparición y el mantenimiento de diferentes trastornos mentales y físicos. Gran parte del desarrollo de procesos psicopatológicos puede explicarse sobre la base de déficits en la implementación de estrategias efectivas de regulación emocional (Garnefski \& Kraaij, 2006; Medrano, \& Trógolo, 2014; Omran, 2011; Zhu et al., 2008).

Aunque existen múltiples maneras de regular las emociones, los procesos cognitivos implicados durante un episodio emocional ejercerían un rol de marcada importancia (Garnefski, \& Kraaij, 2007). En efecto, algunos procesos cognitivos como la rumiación, y la catastrofización, pueden contribuir a que el estado emocional displacentero aumente y se perpetúe, mientras que otras como la reinterpretación o la focalización positiva pueden disminuir el estado displacentero e incluso modificar la valencia de la emoción.

El Cognitive Emotional Regulation Questionnarie (CERQ) fue desarrollado para medir el componente cognitivo de la regulación emocional (Garnefski, Kraaij, \& Spinhoven, 2002; Garnefski, \& Kraaij, 2007). Evalúa nueve estrategias cognitivas de regulación emocional: Rumación, Catastrofización, Autoculparse, Culpar a otros, Poner en Perspectiva, Aceptación, Focalización Positiva, Reinterpretación Positiva, y Refocalización en los Planes (Garnefski \& Kraaij, 2007), y cuenta con estudios psicométricos en diferentes países.

El CERQ ha sido trabajado originalmente en Holanda (Garnefski et al., 2002) en adolescentes y adultos, y ha tenido diferentes adaptaciones: al rumano (Perte \& Miclea, 2011), francés (Jer- mann, Van der Linden, d'Acremont, \& Zermatten, 2006) y español (Domínguez-Sánchez, Lasa-Aristu, Amor, \& Holgado-Tello, 2013) en donde trabajaron con población general; y al chino (Zhu et al., 2008), turco (Tuna \& Bozo, 2012), persa (Abdi, Taban, \& Ghaemian, 2012), y español en Argentina (Medrano, Moretti, Ortiz, \& Pereno, 2013) y Perú (Dominguez \& Medrano, 2016), en los cuales los grupos estudiados fueron de universitarios.

\section{Diferencias culturales en la regulación de emociones}

Las emociones poseen un rol importante en la vida social de las personas y las estrategias de regulación emocional no se encuentran ajenas a la influencia que pueden ejercer los factores socio-culturales. Diferentes trabajos sugieren que estos factores pueden afectar la modulación de las respuestas emocionales (Matsumoto, 2006). Tradicionalmente, el interés por el estudio de las diferencias afectivas entre culturas estuvo centrado en la respuesta emocional, vale decir, un análisis centrado en los factores culturales que llevan a intensificar, disminuir, sustituir o neutralizar la expresión emocional (Ekman et al., 1982). Más recientemente, el interés se ha ampliado al estudio de los procesos implicados en la regulación de una emoción y no sólo en su expresión o respuesta afectiva.

La influencia de los factores socio-culturales sobre los procesos de regulación emocional puede darse en diferentes etapas del proceso emocional. Siguiendo el modelo de Gross (2001), los factores socio-culturales pueden influir sobre los estímulos emocionales a los que se expone una persona, la atención que se presta a dichos estímulos, la manera en la cual se procesan, y finalmente la forma en que debe expresarse la respuesta emocional. De este modo, la cosmovisión, ideología y valores que promueve cada cultura afectaría, al menos en parte, la manera en que las personas procesan los estímulos emocionales (Matsumoto, 2006).

Los factores socio-culturales influyen en las estrategias de regulación emocional a través de múltiples vías. Para algunos autores esta influencia se debería a que las diferentes culturas promueven estrategias de regulación emocional más compatibles con sus 
ideologías y valores (Matsumoto, 2006). Por ejemplo, las estrategias de supresión emocional suelen ser más reforzadas y compatibles con culturas que privilegian los valores colectivos por sobre los individuales. Tal es caso de las culturas confucianas quienes consideran que expresar afecto puede alterar el orden social al priorizar un sentimiento individual por encima de uno colectivo (Keller \& Otto, 2009); en vez de ello promueven el uso de estrategias de regulación centradas en la aceptación emocional (Hanson \& Mendius, 2011). Otra vía de influencia de los factores socio-culturales sobre la regulación de emociones se daría a través de los patrones de socialización y el modelado de los padres (Friedlmeier $\&$ Trommsdorff, 1999). En este sentido puede que los padres lleven a cabo un refuerzo diferencial de ciertas estrategias de regulación emocional en sus hijos. Asimismo los padres pueden influir en el tipo de información que se recibe y en la forma en la que se valora e integra dicha información.

\section{Comparación intercultural de las Estrategias Cognitivas de Regulación Emocional: Invarianza Perú-Argentina}

La invarianza factorial es un aspecto importante a tener en consideración cuando se comparan grupos de sexo, edad o cultura. Esto ayuda a cerciorarse de que las puntuaciones de los grupos comparados en el constructo evaluado presentan un significado similar para cada uno de éstos (Inglés et al., 2009; Inglés, Marzo, Hidalgo, Zhou, \& García-Fernández, 2008). De esta manera, la comparación de los resultados de un test en diferentes grupos solo es admisible si se justifica empíricamente su equivalencia formal y sustantiva (Elosua, 2005). En cambio, si no se llega a establecer la invarianza factorial, cualquier interpretación en torno a las diferencias observadas puede ser errada, ya que las diferencias pueden originarse en fuentes desconocidas y no por las diferencias "verdaderas" en el constructo evaluado (Pedraza \& Mungas, 2008). Es decir, para poder comparar dos grupos se necesita que el test pueda medir constructos idénticos con la misma estructura a través de diferentes grupos (Van der Schoot, Lugtig, \& Hox, 2012).
Si bien se han analizado las diferencias entre países en cuanto a las estrategias cognitivas de regulación de las emociones en algunos estudios usando el CERQ (Domínguez-Sánchez et al., 2013; Zhu et al., 2008) u otros instrumentos que se basan en la teoría de Gross (Haga, Kraft, \& Corby, 2009), no se han encontrado trabajos previos de invarianza factorial entre países que acrediten dichas comparaciones a fin de explorar las similitudes o diferencias interculturales en las regulación cognitiva de las emociones. Si bien se acostumbra comparar culturas cuyos valores distan mucho entre sí (e.g., sociedades individualistas vs. sociedades colectivistas), también se justifica, en vista de los argumentos presentados en párrafos anteriores, comparar culturas de características similares debido a que tienen distintas normas y valores (Pisitsungkagarn \& Busayaprateep, 2013) que potencialmente afectarán la elección de estrategias de regulación de emocional en los individuos que la conforman (Haga et al., 2009). Del mismo modo, es importante conocer las características psicométricas del CERQ a través de las culturas para determinar si la estructura factorial es la misma y si existen diferencias en el constructo estudiado (Wang, Kim, \& Ng, 2012). Por lo tanto, el objetivo de este trabajo fue el estudio de la invarianza factorial del CERQ entre universitarios limeños (Perú) y cordobeses (Argentina), a fin de realizar una comparación intercultural de las estrategias cognitivas de regulación emocional.

\section{Método}

\section{Participantes}

La muestra peruana estuvo constituida por 345 estudiantes universitarios limeños de ambos sexos (varones $=26.4 \%$; mujeres $=73.6 \%$ ), con edades comprendidas entre los 16 y los 62 años $(\mathrm{M}=23.17$ años; $D E=9.28$ ). Por su parte, la muestra argentina se conformó por 358 estudiantes universitarios de la ciudad de Córdoba tanto varones (49.7\%) como mujeres (49.7\%), dejando de reportar ese datos dos estudiantes, con edades comprendidas entre 17 y 61 años $(M=24.62$ años; $D E=10)$. Ambos grupos fueron seleccionados a partir de un muestreo no proba- 
bilístico de tipo accidental, y los mismos accedieron voluntariamente a participar en la investigación.

\section{Instrumentos}

Cognitive Emotional Regulation Questionnarie (CERQ), que fue elaborado por Garnefski y su equipo (Garnefski et al, 2002; Garnefski \& Kraaij, 2007). Consta de 36 ítems, cada uno de los cuales presenta cinco opciones de respuesta que va desde casi nunca (1) hasta casi siempre (5). Evalúa nueve estrategias cognitivas como: Rumiación, Catastrofización, Auto-culparse, Culpar a Otros, Poner en Perspectiva, Aceptación, Focalización Positiva, Reinterpretación Positiva, y Refocalización en los Planes, cada una de las cuales presenta cuatro ítems. El CERQ cuenta con propiedades psicométricas satisfactorias tanto en estudiantes limeños (Dominguez $\&$ Medrano, 2016) como cordobeses (Medrano et al., 2013). Cabe mencionar que como resultado de esos estudios, el ítem 25 ("Pienso que no puedo cambiar nada al respecto") perteneciente a la estrategia Aceptación fue removido.

\section{Procedimiento y análisis de datos}

Para realizar el presente estudio en primer lugar se solicitó el permiso correspondiente a las autoridades universitarias competentes y posteriormente a los docentes para realizar las evaluaciones en el horario habitual de clases. Una vez que los participantes brindaron su consentimiento informado se procedió a administrar los instrumentos.

En primera instancia se efectuó un análisis descriptivo de los ítems y se evaluó el cumplimiento de los supuestos estadísticos de normalidad (univariada y multivariada), linealidad de las relaciones, y ausencia de multicolinealidad. Asimismo se evaluó la existencia de casos atípicos (univariados y multivariados) y el patrón de valores perdidos, con el objeto de verificar que estos no distorsionen los análisis estadísticos.

Para evaluar la invarianza de las estrategias de regulación emocional evaluadas mediante el CERQ entre estudiantes limeños y cordobeses se aplicó la metodología SEM (Structural Equation Modeling), mediante el Análisis Factorial Confirmatorio de Grupo Múltiple utilizando el programa EQS 6.1 (Bentler \& Wu, 2004). Se utilizó el método de máxima verosimilitud (MV), ya que aún con variables con distribución asimétrica existe evidencia de que es un método adecuado para obtener cargas factoriales (Beaducel \& Herzberg, 2006). Los análisis se realizaron sobre la matriz de correlaciones policóricas (Lee, Poon, \& Bentler, 1995), las cuales estiman las variables continuas subyacentes a los ítems de naturaleza ordinal (Bentler, 2010; Dominguez, 2014b), dado además la esperable falta de normalidad estadística de sus ítems (Lei \& Wu, 2012) y el número de frecuencias cero entre pares de ítems (Lei \& Wu, 2012; Savalei, 2011).

Tomando en consideración que el CERQ cuenta con estudios previos que delimitan con claridad la estructura factorial subyacente en muestras peruanas (Dominguez \& Medrano, 2016) y argentinas (Medrano et al., 2013), se evaluó de forma directa la invarianza factorial de medida. Concretamente se efectuó una evaluación progresiva (Byrne, 2008; Elosua, 2005) considerando la invarianza configural (el ajuste en ambas muestras sin añadir ninguna restricción al modelo), la invarianza métrica (invarianza en las cargas factoriales; $\lambda$ ), fuerte (invarianza en las cargas factoriales e interceptos; $\tau_{i}$ ) y estricta (invarianza en las cargas factoriales, interceptos, covarianzas y varianzas de error; $\varepsilon_{i}$ ).

En cuanto a la evaluación de la invarianza configural se usaron los indicadores de ajuste más frecuentes que recomienda la literatura (Dominguez, 2014a; Ferrando \& Anguiano-Carrasco, 2010; Ruiz, Pardo, \& San Martín, 2010; Schreiber, Stage, King, Nora, \& Barlow, 2006). Por lo tanto, se trabajó con el Índice de Aproximación de la Raíz de Cuadrados Medios del Error (RMSEA $\leq$ 0.05; Steiger \& Lind, 1980) y el Índice de Ajuste Comparativo (CFI $\geq$ 0.95; Bentler, 1990). La prueba general de bondad de ajuste $\chi^{2}$ fue calculada aplicándose un ajuste por el efecto de la falta de normalidad de las variables (Satorra \& Bentler, 1994; SB- 2 2).

Para la evaluación de la invarianza métrica, fuerte y estricta, se atendió al cambio significativo en el coeficiente SB- $\chi 2$, y además se utilizó como criterio adicional que el cambio en el coeficiente CFI no fuese superior a 0.01 (Cheung \& Rensvold, 2002). 
Este último criterio es recomendado atendiendo a la sensibilidad de $\chi^{2}$ al tamaño muestral y a la no normalidad (Barrera-Barrera, Navarro-García, \& Peris-Ortiz, 2015).

Algunas de estas restricciones fueron relajadas durante el análisis (Boomsma, 2000), por lo cual se hicieron re-especificaciones que mejoraran el ajuste dentro de un marco de modelamiento a posteriori a fin de poder encontrar identificar los ítems no invariantes en cada una de las etapas evaluadas y así analizar las diversas fuentes de no invarianza. Estas se hicieron primero con base estadística, verificando la significación estadística de los índices de Lagrange IL (Sörbom, 1989), considerando que un $p<0.05$ haría referencia a que la restricción impuesta debe ser relajada para mejorar el ajuste, pero principalmente examinando la racionalidad teórica de los mismos (Boomsma, 2000; Lei \& Wu, 2012).

\section{Resultados}

\section{Análisis descriptivo}

En el análisis exploratorio de datos todos los ítems presentaron una distribución cercana a la normali- dad con valores de asimetría y curtosis univariadas inferiores a \pm 1.5 (Pérez, \& Medrano, 2010). También se evaluó la normalidad multivariada mediante el índice de Mardia obteniéndose valores de 162.37 para los ítems en la muestra argentina y de 182.40 para los ítems en la muestra peruana. Al tratarse de valores superiores a 70 que podrían afectar las estimaciones del modelo (Rodríguez \& Ruiz, 2008) se optó por utilizar la corrección SB- $\chi$. Finalmente se verificó la ausencia de multicolinealidad entre los ítems al no observarse valores superiores a $r=0.90$ entre los ítems en ambas matrices de correlaciones, de la muestra limeña y cordobesa.

\section{Análisis de invarianza}

Para determinar si el modelo original se mantiene invariante tanto en estudiantes limeños como cordobeses, se procedió a evaluar progresivamente la invarianza factorial (tabla 1). Luego de verificar la invariancia configural (M1), no pudo corroborarse la invarianza métrica (M2), se evidenciaron diferencias estadísticas significativas en cuanto a las cargas factoriales (tabla 2). Cuatro ítems se mostraron no-invariantes según los IL $(p<0.05)$, lo cual sugie-

\section{TABLA 1}

Invarianza de medición del CERQ

\begin{tabular}{cccccc}
\hline Modelo & SB- $\chi 2(\mathrm{gl})$ & $\Delta$ SB $-\chi 2(\Delta \mathrm{gl})$ & $p$ & CFI $(\Delta \mathrm{CFI})$ & RMSEA (IC 90\%) \\
\hline M1 & $1816.6332(1048)$ & & & 0.944 & $0.046(0.042,0.049)$ \\
M2 & $1876.5434(1083)$ & $59.91(35)$ & 0.006 & $0.942(0.002)$ & $0.046(0.042,0.049)$ \\
M2a & $1852.2208(1079)$ & $35.59(31)$ & 0.261 & $0.944(0.000)$ & $0.045(0.042,0.049)$ \\
M3 & $2558.3489(975)$ & $706.13(104)$ & 0.000 & $0.743(0.201)$ & $0.066(0.063,0.069)$ \\
\hline
\end{tabular}

Nota. M1: Configural; M2: Métrica; M2 Métrica parcial (sin restricciones de $\lambda i$ sobre 12, 17, 29 y 31); M3: Fuerte.

Fuente: elaboración propia

TABLA 2

Ítems del CERQ con no-invarianza métrica

\begin{tabular}{lccc}
\hline \multicolumn{1}{c}{ Ítem } & Factor & $\lambda$ Argentina & $\lambda$ Perú \\
\hline 12.- Pienso que puedo convertirme en una persona más fuerte & Reinterpretación positiva & 0.52 & 0.65 \\
17.- Siento que soy el responsable de lo que ha pasado & Autoculparse & 0.71 & 0.48 \\
29. - Pienso que otros son responsables de lo que ha ocurrido & Culpar a otros & 0.85 & 0.75 \\
31.- Miro las partes positivas del problema & Reinterpretación positiva & 0.88 & 0.74 \\
\hline
\end{tabular}

Fuente: elaboración propia 
re la posibilidad de una invarianza parcial más que completa (Byrne, 2008). En tal sentido, se liberaron esos parámetros a fin de probar la invarianza métrica parcial ( $\mathrm{M}_{2}$; se relajaron las restricciones a las cargas factoriales de los cuatro ítems no-invariantes), y se obtuvo un cambio no significativo en el SB- $\chi^{2} \mathrm{ni}$ en el CFI con respecto al M1. Finalmente, sobre la base del modelo $\mathrm{M}_{\mathrm{a}}$ se probó la invarianza fuerte, pero esta no obtuvo el respaldo de los índices de ajuste (tabla 1), se apreciaron gran cantidad de interceptos que diferían significativamente entre ambos grupos (tabla 3).

\section{Discusión}

El objetivo del trabajo fue determinar si existen diferencias interculturales en cuanto a las estrategias cognitivas de regulación emocional entre estudiantes limeños y cordobeses, a través del estudio progresivo de la invarianza factorial entre los grupos. Cabe mencionar que no existen estudios previos de invarianza factorial a fin de estudiar di- ferencias interculturales, siendo el más cercano el desarrollado en China (Zhu et al., 2008), aunque es de carácter descriptivo y no contempla el uso de análisis de invarianza utilizando metodología SEM. Del mismo modo, otras investigaciones (e.g., Domínguez-Sánchez et al., 2013) presentan conclusiones de índole intercultural con base en las comparaciones de resultados de diversos estudios. En tal sentido, esta es la primera investigación sobre diferencias interculturales llevada a cabo bajo un estudio progresivo de la invarianza factorial utilizando metodología SEM.

En cuanto a la invarianza configural cabe destacar que se verifica la estructura de nueve factores oblicuos, lo cual es congruente con los estudios antecesores (Domínguez-Sánchez et al., 2013; Garnefski et al, 2002; Medrano et al, 2013). Aparentemente las diferentes estrategias cognitivas de regulación emocional podrían agruparse en nueve factores en diferentes culturas. Este aspecto es fundamental cuando se efectúan investigaciones de carácter transcultural, ya que podría existir

\section{TABLA 3}

Interceptos de los Ítems no invariantes del CERQ: invarianza fuerte

\begin{tabular}{|c|c|c|c|}
\hline Ítem & Dimensión & $\tau$ Argentina & $\tau$ Perú \\
\hline 9.- Pienso que otros son culpables de lo que me pasó & Culpar a otros & 0.739 & 1.24 \\
\hline $\begin{array}{l}\text { 21.- Pienso en los fallos que otros han cometido en esa situa- } \\
\text { ción }\end{array}$ & Culpar a otros & 1.983 & 2.529 \\
\hline 36.- Pienso que fundamentalmente la causa recae en otros & Culpar a otros & 0.983 & 1.709 \\
\hline 26.- Pienso en los errores que he cometido & Autoculparse & 2.719 & 2.415 \\
\hline $\begin{array}{l}\text { 33.- Pienso que fundamentalmente la causa recae en mí mis- } \\
\text { mo }\end{array}$ & Autoculparse & 2.016 & 2.091 \\
\hline $\begin{array}{l}\text { 15.- Estoy preocupado por lo que siento y pienso acerca de lo } \\
\text { que me ha pasado }\end{array}$ & Rumiación & 1.623 & 1.606 \\
\hline $\begin{array}{l}\text { 22.- Normalmente pienso que lo que me ha pasado a mí es lo } \\
\text { peor que le puede pasar a alguien }\end{array}$ & Catastrofización & 0.874 & 1.165 \\
\hline 7.- Pienso que podría haber sido mucho peor & Poner en perspectiva & 2.000 & 1.221 \\
\hline 13.- Pienso cómo puedo afrontar mejor la situación & Focalización en los planes & 1.277 & 1.335 \\
\hline 19.- Pienso en cómo cambiar la situación & Focalización en los planes & 0.782 & 0.402 \\
\hline 30.- Pienso un plan para que en un futuro me vaya mejor & Focalización en los planes & 0.874 & 0.909 \\
\hline 4.- Pienso en cosas más agradables que lo que me ha pasado & Focalización positiva & 2.425 & 2.439 \\
\hline
\end{tabular}

Fuente: elaboración propia 
un sesgo de constructo que no permita una correcta interpretación de los resultados. Asimismo, asumir que el constructo se comporta de manera semejante puede llevar a extrapolar modelos no ajustados a las características y especificidades de cada cultura. En función de los resultados obtenidos puede señalarse que el modelo de nueve factores de regulación cognitiva es un modelo sólido y robusto de las diferencias culturales existentes entre Argentina y Perú.

Por otra parte es importante señalar que se mantienen invariantes ciertas propiedades métricas. Tanto las cargas factoriales como los interceptos de la mayor parte de ítems se mostraron invariantes. Concretamente se verificó la invarianza de las estrategias de Aceptación, Poner en perspectiva, y Focalización positiva. No obstante debe considerarse que se trata de estrategias que involucran un procesamiento más racional y complejo de la información, por lo que las semejanzas en el nivel educativo de los participantes (universitarios en ambas culturas) podrían estar moderando las diferencias socio-culturales entre los grupos. Sería provechoso replicar los análisis incluyendo además participantes con bajo nivel educativo.

Un panorama similar se observa con las estrategias Rumiación y Catastrofización que se mantienen invariantes. Cabe señalar que la invarianza era esperable en estas dimensiones ya que se trata de estrategias automáticas de regulación emocional que poseen una mayor impronta biológica y en menor medida cultural. En efecto, tal como plantea Medrano (2014), estas estrategias constituyen resabios evolutivos destinados a detectar amenazas y reaccionar automáticamente para aumentar la seguridad y supervivencia del organismo. Al ser estrategias más antiguas en términos evolutivos se encontrarían asentadas en estructuras subcorticales, por tanto menos afectadas por factores socio-culturales. No obstante se requiere de mayores estudios para corroborar este planteamiento.

Otro aspecto a contemplar es que las culturas comparadas son semejantes y próximas entre sí. Dicha proximidad no está dada solo por factores geográficos, sino también por componentes socioculturales. De hecho, algunas culturas aborígenes se extendieron en ambos territorios, y los procesos coloniales y migratorios fueron muy semejantes en los dos países. Al existir tantas proximidades culturales es esperable que el modelo se comporte de forma invariante.

No obstante, un análisis pormenorizado y más restrictivo permite identificar un funcionamiento no-invariante en algunos reactivos e interceptos. De acuerdo con los resultados obtenidos los estudiantes limeños y cordobeses presentan diferencias en las estrategias Culpar a otros y Autoculparse, siendo los limeños personas que en su mayoría se caracterizan más por culpar a los demás, mientras que los estudiantes de Córdoba prefieren tomar toda la responsabilidad cuando ocurre un evento displacentero. Asimismo, los estudiantes limeños sobresalen en la estrategia Focalización en los planes, es decir, en elaborar algún tipo de solución para que a futuro se pueda afrontar de forma más eficiente un problema similar.

Se deberían realizar estudios más profundos para determinar el origen de estas diferencias. Tal como se planteó inicialmente, los factores socio-culturales pueden influir en las estrategias de regulación emocional a través de múltiples vías. Las evidencias recabadas en el presente estudio no son suficientes para elaborar un modelo explicativo de las diferencias observadas, sería de interés indagar en un futuro estudio si el uso de estas estrategias se relaciona con especificidades en las ideologías y valores de cada cultura.

\section{Referencias}

Abdi, S., Taban, S., \& Ghaemian, A., (2012). Cognitive emotion regulation questionnaire: Validity and reliability of the Persian traslation of the CERQ (36-item). Procedia, Social and Behavioral Sciences, 32, 2-7.

Barrera-Barrera, R., Navarro-García, A., \& Peris-Ortiz, M. (2015). El papel de la invarianza factorial en la validación del constructo calidad de servicio electrónico. Revista Europea de Dirección y Economía de la Empresa, 24(1), 13-24.

Bentler, P. M. (1990). Comparative fit indexes in structural models. Psychological Bulletin, 107, 238-246. 
Bentler, P. M. (2010). SEM with simplicity and accuracy. Journal of Consumer Psychology, 20, 215-220.

Bentler, R. M., \& Wu, E. J. C. (2004). EQS 6.1 for windows [Statistical Program]. Encino, CA: Multivariate Software, Inc.

Beaducel, A., \& Herzberg, P. Y. (2006). On the performance of maximum likelihood versus means and variance adjusted weighted least squares estimation in CFA. Structural Equation Modeling: A Multidisciplinary Journal, 13(29), 186- 203.

Boomsma, A. (2000). Reporting analyses of covariance structures. Structural Equation Modeling, 7(3), 461-483.

Byrne, B. M. (2008).Testing for multigroup equivalence of a measuring instrument: A walk through the process. Psicothema, 20(4), 872-882.

Cheung, G., \& Rensvold, R. (2002). Evaluating goodness-of-fit indexes for testing measurement invariance. Structural Equation Modeling, 9, 233-255.

Dominguez, S. (2014a). Análisis Psicométrico de la Escala de Bienestar Psicológico para Adultos en estudiantes universitarios de Lima: un enfoque de ecuaciones estructurales. Psychologia: Avances en la disciplina, 8(1), 23-31.

Dominguez, S. (2014b). iMatrices Policóricas/Tetracóricas o Matrices Pearson? Un estudio metodológico. Revista Argentina de Ciencias del Comportamiento, 6(1), 39-48.

Dominguez, S., \& Medrano, L. (2016). Propiedades psicométricas del Cognitive Emotional Regulation Questionnarie (CERQ) en estudiantes universitarios de Lima. Psychologia: Avances en la disciplina, 10(1), 53-67.

Domínguez-Sánchez, F., Lasa-Aristu, A., Amor, P., \& Holgado-Tello, F. (2013). Psychometric properties of the spanish version of the Cognitive Emotion Regulation Questionnaire. Assessment, 20(2), 253-261.

Ekman, P., Friesen, W. V., O'Sullivan, M., Chan, A., Diacoyanni-Tarlatzis, I., Heider, K., ... \& Tzavaras, A. (1987). Universals and cultural differences in the judgments of facial expressions of emotion. Journal of personality and social psychology, 53(4), 712-717.

Elosua, P. (2005). Evaluación progresiva de la invarianza factorial entre las versiones original y adaptada de una escala de autoconcepto. Psicothema, 17(2), 356-362.

Ferrando, P. \& Anguiano, C. (2010). El análisis factorial como técnica de investigación en psicología. En Papeles del psicólogo, 31(1), 18-33.

Friedlmeier, W., \& Trommsdorff, G. (1999). Emotion regulation in early childhood: A cross-cultural comparison between german and japanese toddlers. Journal of Cross-cultural Psychology, 30(6), 684-711.

Garnefsky, N., \& Kraaij, V. (2006). Relationship between cognitive emotion regulation strategies and depressive symptoms: A comparative study of five specific samples). Personality and Individual Differences, 40, 1659, 1669.

Garnefsky, N., \& Kraaij, V. (2007). The Cognitive Emotion Regulation Questionnarie: Psychometric features and prospective relationships with depression and anxiety in adults. European Journal of Psychological Assessment, 23(3), 141-149. Garnefsky, N., Kraaij, V., \& Spinhoven, P. (2001). Negative life events, cognitive emotion regulation and emotional problems. Personality and Individual Differences, 30, 1311-1327.

Garnefsky, N., Kraaij, V., \& Spinhoven, P. (2002). Manual for the use of Cognitive Emotion Regulation Questionnarie. Leiderdorp, the Netherlands: DATEC. Gross, J. (1998). The emerging field of the emotion regulation: an integrative review. Review of General Psychology, 2(3), 271-299.

Gross, J. (2001). Emotion regulation in adulthoof: Timing is everithing. Current Direction in Psychological Science, 10, 214-219.

Gross, J. \& Thompson, R. (2007). Emotion Regulation: Conceptual foundations. En J. Gross (Ed.), Handbook of emotion regulation (pp. 3-24). New York: Guilford Press.

Haga, S., Kraft, P., \& Corby, E. (2009). Emotio regulation: Antecedents and well-being outcomes of cognitive reappraisal and expressive supression in cross-cultural samples. Journal of Happiness Studies, 10, 271-291.

Hanson, R., \& Mendius, R. (2011). El cerebro de Buda. La neurociencia de la felicidad, el amor y la sabiduría. Barcelona: Mil razones. 
Inglés, C., Benavides, G., Redondo, J., García-Fernández, J., Ruiz-Esteban, C., Estevez, C., \& Huescar (2009). Conducta prosocial y rendimiento académico en estudiantes españoles de Educación Secundaria Obligatoria. Anales de Psicología, 25, 93-101.

Inglés, C., Marzo, J., Hidalgo, M., Zhou, X., \& García-Fernández, J. (2008). Factorial invariance of the Questionnarie about Interpersonal Difficulties for Adolescents across spanish and chinese adolescent samples. Measurement and Evaluation in Counseling and Development, 41, 89-103.

Jermann, F., Van der Linden, M., d’Acremont, M., \& Zermatten, A. (2006). Cognitive Emotion Regulation Questionnarie (CERQ): Confirmatory factor analysis and psychometric properties of the french traslation. European Journal of Psychological Assessment, 22(2), 126-131.

Keller, H., \& Otto, H. (2009). The cultural socialization of emotion regulation during infancy. Journal of Cross-cultural Psychology, 40(6), 996-1011.

Koole, S. (2009). The psychology of the emotion regulation: an integrative review. Cognition and emotion, 23(1), 4-41.

Lee, S.-Y., Poon, W.Y. \& Bentler, P. M. (1995). A twostage estimation of structural equation models with continuous and polytomous variables. British Journal of Mathematical and Statistical Psychology, 48, 339-358.

Lei, P-W. \& Wu, Q. (2012). Estimation in structural equation modeling. In: Hoyle, R. H. (Ed.), Handbook of structural equation modeling (pp. 164-179). New York: Guildford Press.

Matsumoto, D. (2006). Are cultural differences in emotion regulation mediated by personality traits? Journal of Cross-cultural Psychology, 37(4), 421-437.

Medrano, L. (2014). Cognición y Emoción: el papel de los Procesos Cognitivos en la Regulación de Emociones. Conferencia presentada en el V Congreso Iberoamericano y XI Congreso Nacional de Profesionales y Estudiantes de Psicología. Lima, Perú.

Medrano, L., \& Trógolo, M. (2014). Validación de la escala de dificultades en la regulación emocional en la población universitaria de Córdoba, Argentina. Universitas Psychologica, 13(4), 1345-1356.

Medrano, L., Moretti, L., Ortiz, A., \& Pereno, G. (2013). Validación del Cuestionario de Regulación Emo- cional Cognitiva en Universitarios de Córdoba, Argentina. Psykhe, 22(1), 83-96.

Omran, M. (2011). Relationship between cognitive emotion regulation strategies with depression and anxiety. Open Journal of Psychiatry, 1, 106-109.

Pedraza, O., \& Mungas, D. (2008). Measurement in cross-cultural neuropsychology. Neuropsychology Review, 18, 184-193.

Perte, A., \& Miclea, M. (2011). The standarization of the Cognitive Emotion Regulation Questionnaire (CERQ) on romanian population. Cognition, Brain, Behavior. An Interdisciplinary Journal, 15(1), 111-130.

Pérez, E., \& Medrano, L. (2010). Análisis factorial exploratorio: bases conceptuales y metodológicas. Revista Argentina de Ciencias del Comportamiento, 2(1), 58-66.

Pisitsungkagarn, K., \& Busayaprateep, P. (2013). Emotion regulation: A critical review of cross-cultural validity of emotion suppression. Paper presented at The Asian Conference on Psychology \& the Behavioral Sciences. Recuperado de http://iafor.org/archives/ offprints/acp2013-offprints/ACP2013_0247.pdf

Rodríguez, M., \& Ruiz, M. (2008). Atenuación de la asimetría y de la curtosis de las puntuaciones observadas mediante transformaciones de variables: Incidencia sobre la estructura factorial. Psicológica, 29, 205-227.

Ruiz, M., Pardo, A., \& San Martín, R. (2010). Modelos de Ecuaciones Estructurales. Papeles del Psicólogo, 31(1), 34-45.

Satorra, A., \& Bentler, P. M. (1994). Corrections to test statistics and standard errors in covariance structure analysis. In A. von Eye \& C. C. Clogg (Eds.), Latent variables analysis: Applications for developmental research (pp. 399-419). Thousand Oaks, CA: Sage.

Savalei, V. (2011). What to do about zero frequency cells when estimating polychoric correlations. Structural Equation Modeling: A Multidisciplinary Journal, 18(2), 253-273.

Schreiber, J., Stage, F., King, J., Nora, A., \& Barlow, E. (2006). Reporting structural equation modeling and confirmatory factor analysis results: a review. The Journal of Education Research, 99(6), 323-337. 
Sörbom, D. (1989). Model modification. Psychometrika, 54, 371-384.

Soto, J., Perez, C., Kim, Y., Lee, E., \& Minnick, M. (2011). Is expressive suppression always associated with poorer psychological functioning? A crosscultural comparison between European Americans and Hong Kong Chinese. Emotion, 11(6), 1450-1455.

Steiger, J. H., \& Lind, J. C. (1980, May). Statistically based tests for the number of common factors. Paper presented at the annual meeting of the Psychometric Society, Iowa City, IA.

Tuna, E., \& Bozo, O. (2012). The Cognitive Emotion Regulation Questionnarie: Factor structure and psychometric properties of the Turkish version.
Journal of Psychopathology and Behavioral Assessment, 34, 564-570.

Van der Schoot, R., Lugtig, P., \& Hox, J. (2012). A checklist for testing measurement invariance. European Journal of Developmental Psychology 9(4), 486-492.Wang, C., Kim, D., \& Ng, K. (2012). Factorial and item-level invariance of an Emotional Intelligence Scale across groups of international students. Journal of Psychoeducational Assessment, 30(2), 160-170.

Zhu, X., Auerbach, R., Yao, S., Abela, J., Xiao, J., \& Tong, X. (2008). Psychometric properties of the Cognitive Emotion Regulation Questionnarie: Chinese versión. Cognition and Emotion, 22(2), 288-307. 